\title{
Relativistic and non-relativistic studies of nuclear matter
}

\author{
Manoj K. Banerjee ${ }^{1}$ and John A. Tjon ${ }^{1,2}$ \\ 1 Department of Physics, University of Maryland, \\ College Park, MD, USA, 20742-4111 \\ ${ }^{2}$ Institute for Theoretical Physics, University of Utrecht \\ 3508 TA Utrecht, The Netherlands
}

(November 5, 2018)

\begin{abstract}
Recently we showed that while the tensor force plays an important role in nuclear matter saturation in non-relativistic studies, it does not do so in relativistic studies. The reason behind this is the role of $M^{*}$, the sum of nucleon mass and its attractive self-energy in nuclear matter. Yet nonrelativistic calculations at a certain level of approximation are far less difficult than comparative relativistic calculation. Naturally the question arises if one can modify a nonrelativistic method, say, the lowest order Brueckner theory (LOBT), to reproduce approximately the results of a relativistic calculation. While a many body effect, the role of $M^{*}$ is intrinsically relativistic. It cannot be simulated by adding multi-body forces in a nonrelativistic calculation. Instead, we examine if adding a set of recipes to LOBT can be useful for the purpose. We point out that the differences in the results arise principally from two reasons - first, the role of $M^{*}$ and second, the disappearance in a relativistic treatment of the gap in the hole and particle energy spectra, present in LOBT. In this paper we show that LOBT, modified by recipes to remove these two reasons, generates results quite close to those of Dirac-Brueckner theory.
\end{abstract}

\section{INTRODUCTION}

In a recent paper [1] we have shown that a relativistic treatment of the saturation mechanism of nuclear matter differs in an essential way from a non-relativistic description. As has already been pointed out by Bethe more than 40 years ago, assuming that the nucleons behave non-relativistically, saturation takes place because the large attractive contribution of second and higher OPEP tensor force is increasingly quenched with increasing density due to Pauli blocking. A careful analysis of the relativistic results of Amorim and Tjon [2] showed that the role of the tensor force in the saturation mechanism is significantly reduced. The saturation occurs mainly due to reduction of the scalar charge with increasing density, a result familiar from mean field treatments of nuclear matter [3].

Simultaneously, it should be stressed that the role of the tensor force in the two-nucleon problems continues to be large in a relativistic treatment exactly as in a non-relativistic treatment. In Ref. [1] we showed that the reduction of the role of the tensor force is due to the role of $M^{*}$, a manifestly many-body effect.

While the issue of saturation mechanism is quite dramatic there are also detailed differences in the results of non-relativistic lowest order Brueckner theory and its relativistic counter part - Dirac-Brueckner theory. 
This apparent shift in the physics of nuclear matter in going from non-relativistic to relativistic description is quite surprising and raises question about the correct treatment of the problem. Because of the precision and sophistication achieved in non-relativistic treatments one cannot justifiably suggest that it be abandoned. In particular, one may attempt to modify these equations to also include some of the salient features of a relativistic analysis. Results of relativistic Dirac-Brueckner theories are closely tied to two features: a self-consistent $M^{*}$ and no gap in the single particle spectrum at the top of the Fermi sea. We examine in this paper the possibility of incorporating these two features in a non-relativistic theory.

\section{MODIFICATION OF THE BRUECKNER-BETHE-GOLDSTONE EQUATIONS}

Starting from the lowest order non-relativistic Brueckner-Bethe-Goldstone (LOBT) equations, one may attempt to incorporate as recipes the previously mentioned features present in the relativistic Dirac-Brueckner analysis. The existence of a gap in the single nucleon spectrum at the Fermi surface depends on the organization of the many-body theory such as - the non-relativistic LOBT or relativistic Dirac-Brueckner (DB) theory. The LOBT is based on Goldstone linked cluster expansion. The propagators between two events are global. They are not single particle propagators as in Feynman perturbation expansion. In LOBT different sets of diagrams are summed in the definition of the hole and the particle energies and, naturally, a gap arises at the Fermi surface.

An alternative approach is based on the Green's function method 沟, which is structurally the same as Feynman perturbation expansion but using non-relativistic physics. Here the hole and the particle energies sum similar sets of self-energy diagrams. As a natural consequence the single particle potential is continuous across the Fermi surface [4], i.e. , there is no gap. In DB the single nucleon energies are also just the self-energies and there is no gap.

The absence or presence of a gap can be built into the 2-nucleon Green's function $S_{2}$ appearing in the Bethe-Goldstone equation for the G-matrix

$$
\left\langle\vec{p}^{\prime}|G| \vec{p}\right\rangle=\left\langle\vec{p}^{\prime}|\underline{\mathrm{V}}| \vec{p}\right\rangle+\sum_{\lambda, i} \int \frac{d^{3} p^{\prime \prime}}{(2 \pi)^{3}}\left\langle\vec{p}^{\prime}|V| \vec{p}^{\prime \prime}\right\rangle S_{2}\left(\vec{p}^{\prime \prime}\right)\left\langle\vec{p}^{\prime \prime}|G| \vec{p}\right\rangle,
$$

where $\lambda, i$ are the helicities and isospin of the intermediate state and $S_{2}$ is the Pauli-blocked 2-nucleon Green's function

$$
S_{2}\left(\vec{p}^{\prime \prime}\right)=\frac{Q_{\text {Pauli }}}{E\left(p^{\prime \prime}\right)-\epsilon(p)}
$$

with $E$ and $\epsilon$ being the single nucleon energy above and below the Fermi momentum $p_{F}$. In standard LOBT

$$
E(p)=\frac{p^{2}}{2 M} ; \epsilon(p)=A+\frac{p^{2}}{2 m^{*}} .
$$

The quantity $A$ and the effective mass $m^{*}$ are usually determined in the LOBT calculations in a self-consistent way.

From a practical point of view, it may be useful to explore the possibility of finding recipes which when incorporated into non-relativistic LOBT reproduce closely the results of Dirac- Brueckner theory. We have 
tried this and the next section presents the results using our recipe of including these effects. All numerical results reported and used in this paper are generated with a modified version of a program written by Gruemmer [6].

\section{LOBT INCLUDING RELATIVIZED $M^{*}$}

The principal features of our method of incorporating the necessary recipes into LOBT are described below.

1. Introduction of $M^{*}$.

The precise meaning of $M^{*}$ in the present context is made clear in Eq (6). Specifically we choose the simple parametrization

$$
M^{*} / M=1-\alpha \rho / \rho_{0},
$$

where $\rho_{0}=0.17 \mathrm{fm}^{-3}$ is the density of normal nuclear matter.

2. Modification of the single nucleon energy spectrum.

We examine two alternatives for the modified LOBT calculation. We choose simple quadratic forms for the single nucleon spectrum below and above the Fermi sea:

$$
\begin{aligned}
& \text { for } p<k_{F}, \epsilon(p)=A+\frac{p^{2}}{2 m^{*}}, \\
& \text { for } p>k_{F}, E(p)=\frac{p^{2}}{2 M^{*}} .
\end{aligned}
$$

With the spectrum as defined above the gap is

$$
\Delta=k_{F}^{2}\left(\frac{1}{2 M^{*}}-\frac{1}{2 m^{*}}\right)-A .
$$

The alternatives we examined are:

(a) No self-consistency, no gap. The method of doing the nonselfconsistent is as follows. One begins by choosing somewhat arbitrarily $m^{*}=M$, calculate the $G$-matrix with it and subsequently the new hole spectrum and the new $m^{*}$ with it and then stop. The absence of a gap is ensured by setting

$$
A=k_{F}^{2}\left(\frac{1}{2 M^{*}}-\frac{1}{2 m^{*}}\right)
$$

(b) self-consistent $\epsilon(p)$, no gap. Here the hole spectrum is obtained self-consistently and then A is fixed with Eq. (8). 


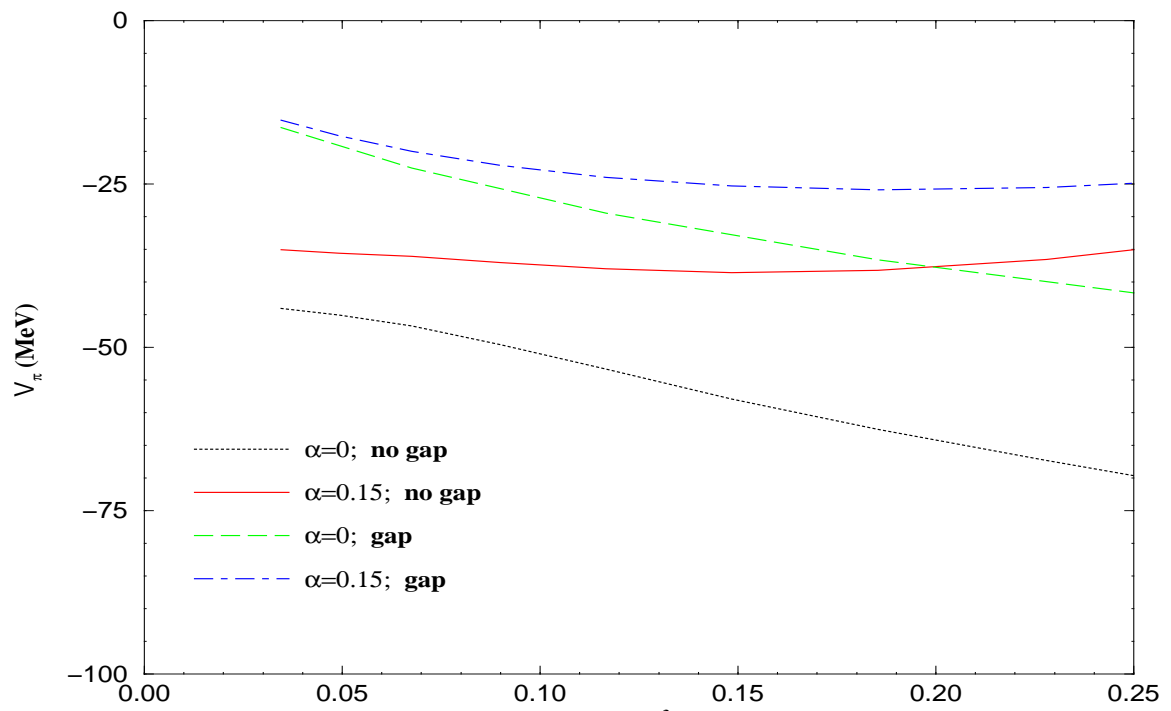

FIG. 1. Plots of $V_{\pi}$ plotted as function of $\rho$ for $\alpha=0 \stackrel{2}{2}\left(\mathbf{f m}_{\alpha}^{-3}\right)=0.15$ and for hole energy spectrum with and without gap.

A few remarks about the status of OPEP are in order. In Ref [1] we have described how $M^{*}<M$ leads to reduction of the role of OPEP. A measure of this role is the quantity

$$
V_{\pi}=\left\langle N M\left|V_{O P E P}\right| N M\right\rangle=g_{\pi}^{2} \frac{d}{d g_{\pi}^{2}} E,
$$

where $|N M\rangle$ is the nuclear matter ground state and $E$ is the ground state energy. In Ref $[1]$ the dependence of $V_{\pi}$ on $M^{*}$ was studied by modifying the value of the scalar self-energy $S=M^{*}-M$ from its self-consistently determined value [2] by replacing $S$ with $\alpha S, 0 \leq \alpha \leq 1$. Here $M^{*}$ is introduced by hand and is defined by Eq. (4). It should be noted that the definition of $\alpha$ here is different from that used in Ref [1], though related in spirit. The reduction of $\left|V_{\pi}\right|$ with decreasing $M^{*}$ has been shown in Fig. 2 of Ref [1]. In Fig. 1] $V_{\pi}$ is plotted as a function of $\rho$ for $\alpha=0$ and $\alpha=0.15$ and for hole energy spectrum with and without gap. We see that the reduction of $\left|V_{\pi}\right|$ with decreasing $M^{*}$ occurs for both choices of the single particle spectrum. The slight upward curvature of $\alpha=0.15$ is due to the contribution of the first order OPEP, being of a repulsive nature and which becomes dominant at higher density.

We note that despite the reduced role in the saturation mechanism the contribution of OPEP to $E / A$ continues to be important in the present treatment, thus duplicating this feature of the relativistic treatment [1].

In Fig. 2 we show the predictions when $\alpha=0$ and assuming that there is no gap present in the single particle spectrum. The curve is obtained by varying $g_{\sigma_{1}}$ parameter in the Bonn-C potential in the range 
between 6.5 and 9.0. The slope found in the case of the nonrelativistic LOBT calculation (filled circles) agrees well with the linear Coester-line. In accordance with [4] we find that dropping the gap in the single particle spectrum leads to a significant shifting towards the empirical result. It is however not sufficient to reproduce the empirical data. This is achieved by including the $M^{*}$ modification. It has the important effect of changing the slope of the found linear dependence. The predictions are shown in Fig. 3 .

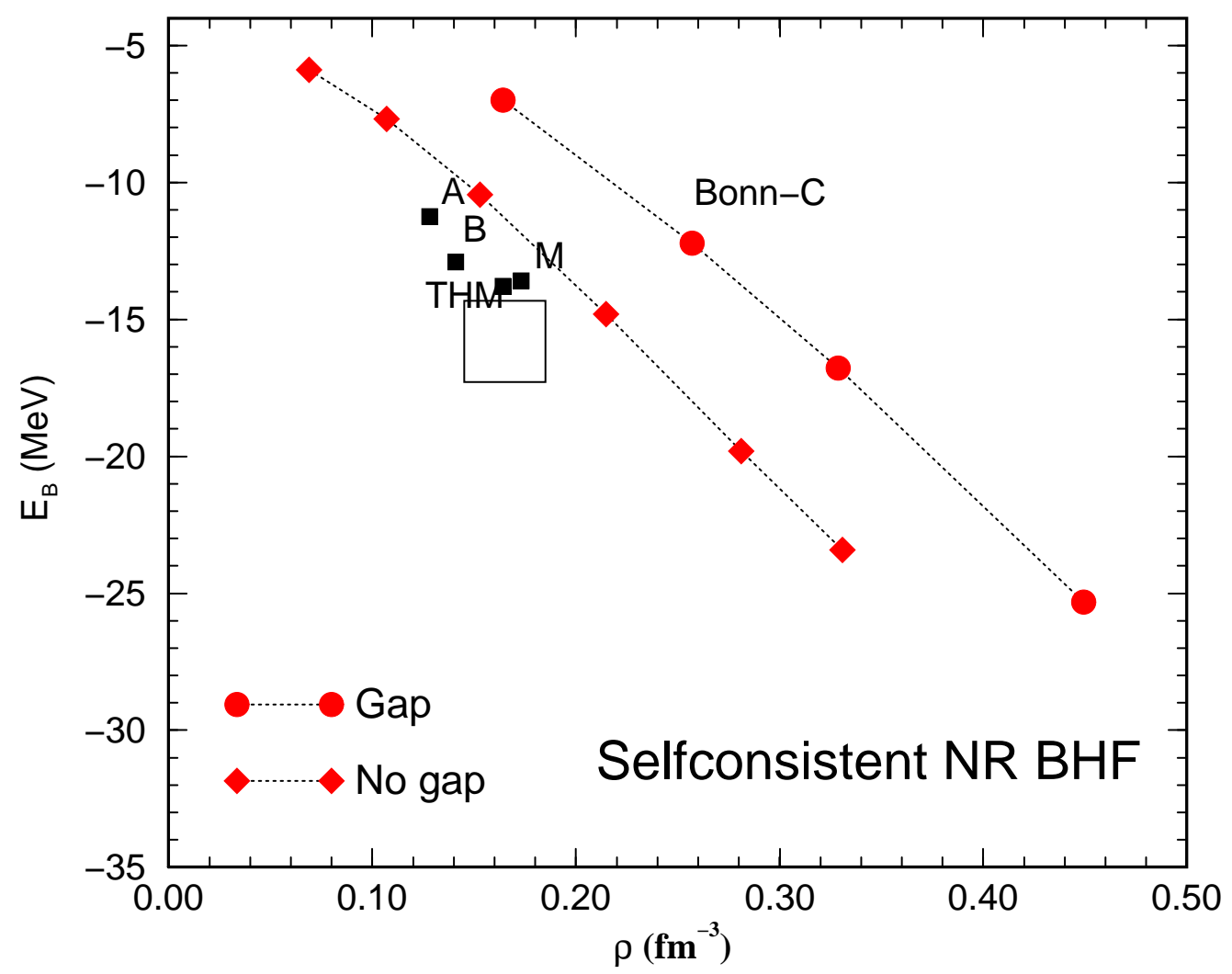

FIG. 2. Predictions of $E_{B}=-E / A$ are shown for a selfconsistent calculation (diamonds), assuming that there is no gap present in the single particle spectrum and no $M^{*}$-modification. Also is shown a selfconsistent nonrelativistic LOBT calculation (circles). The Bonn-C potential of Ref. [7] is used, where the various calculated curves are obtained by varying the $\sigma_{1}$ - coupling constant. For completeness, the relativistic calculations of Refs. [2]:7], labelled as (A, B), $\mathrm{M}$ and THM, are also shown.

The calculations have been carried out using the recipes described earlier. The Bonn-C potential has been used with the original $g_{\sigma_{1}}=8.63$ and where the $M^{*}$-modification is implemented by hand using the $\alpha$-parameter of Eq. (何. The main features to note are as follows: 


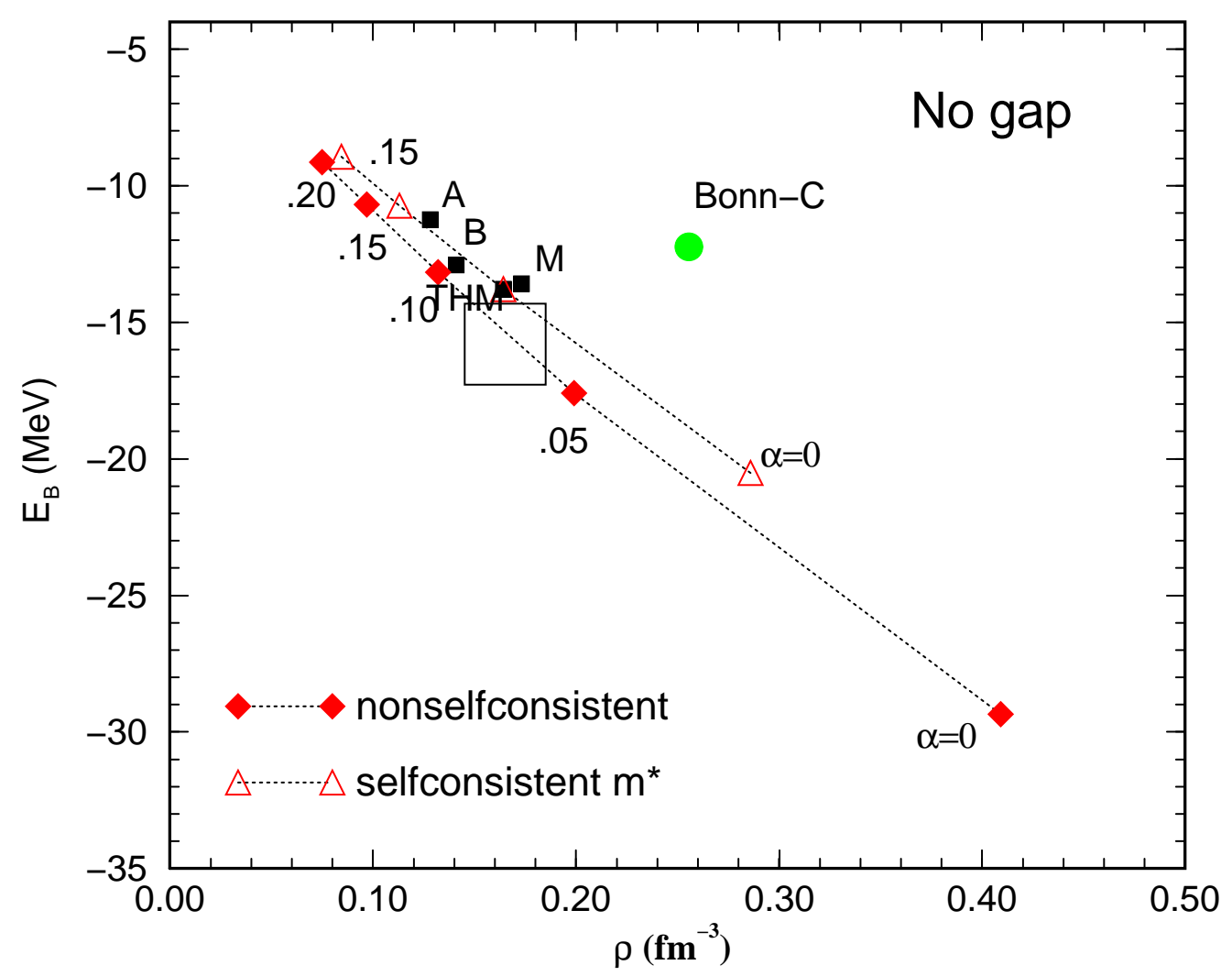

FIG. 3. Predictions of $E_{B}=-E / A$ assuming that there is no gap in the single nucleon spectrum present. The two curves are obtained by varying the $\alpha$-parameter. The results of nonselfconsistent calculations are shown as filled diamonds. Those of selfconsistent calculations are shown as triangles. For completeness the results of nonrelativistic LOBT calculation for BonnC potentials of Ref. [7] and the relativistic calculations of Refs. [2, ], 8], labelled as (A, B), $\mathrm{M}$ and THM, are also shown.

1. Once the gap is removed the results are not all that different for non-selfconsistent and selfconsistent calculations.

2. Use of $\alpha>0$ is critically important to reproduce the empirical data.

3. It is not surprising that the modelling which agrees best with the relativistic results of Refs. [2,7,8] have selfconsistent hole spectrum and no gap. Both features are present in the relativistic Dirac-Brueckner calculations.

4. Finally, we stress that what we have presented are recipes and not theories. The removal of the gap 
is done by hand. The quantity, $M^{*}$ is introduced by hand to simulate the very important feature of relativistic many nucleon problem. It appears that our recipes prefer $\alpha \sim 0.15$, while Ref. [2] finds $\alpha \simeq 0.3$.

\section{CONCLUDING REMARKS}

One expects a non-relativistic theory to be able to join on to a relativistic theory in a smooth fashion. After considerable numerical and analytical examination of the issues we conclude, not too surprisingly, that it is not possible to generate the two features, gap in the single nucleon spectrum and the role of $M^{*}$, in a non-relativistic theory in a natural way. Yet the level of rigor and completeness of non-relativistic nuclear many-body theory has risen well above LOBT. See Ref. [5] for a recent review. The improvements have taken account of many-nucleon interactions in significantly greater detail and precision. In contrast, the relativistic nuclear many-body treatment is essentially at the level of Dirac-Brueckner theory. So there may be considerable reluctance to give up the non-relativistic approach in favor of a relativistic approach. However, the $M^{*}$ effect, which is one of the central issues of this paper, arises because what is energy in non-relativistic physics manifests itself as a sum of Lorentz scalar and Lorentz vector terms in relativistic physics. No amount of improvement in the dynamical aspects of a non-relativistic theory can address this issue. In other words, there is no magic three- or more-body force which, upon inclusion in non-relativistic calculations will imitate the role of $M^{*}$. In any case, if a genuinely new force is introduced in a non-relativistic treatment a similar force must also be be added in the relativistic treatment, unless it is already present. Thus a truly satisfactory resolution of the problem appears to be impossible.

Despite these caveats it is useful, from a practical point of view, to explore the possibility of finding recipes which when incorporated into non-relativistic LOBT reproduce closely the results of Dirac- Brueckner theory. This we have done in the present paper From this study we have found that the presence of a gap, as predicted by the standard LOBT analysis, or absence has an important effect. In addition, the $M^{*}$ modification changes the slope of the nonrelativistic Coester-line significantly, leading to results in accordance with the Dirac-Brueckner analysis. However it should be stressed, that the prescriptions we have used are simply recipes to simulate some of the aspects of the relativistic analysis.

[1] M. K. Banerjee and J. A. Tjon, Phys. Rev C, 58, 2120 (1998)

[2] A. Amorim and J. A. Tjon, Phys. Rev. Lett., 68, 772 (1992)

[3] B. D. Serot and J. D. Walecka, Adv. Nucl. Phys., 16, 1 (1986)

[4] C. Mahaux, Nucl. Phys. A328, 24 (1979) 
[5] V. R. Pandharipande, Int. J. Mod. Phys., B13, 543 (1999)

[6] F. Gruemmer, Private communication

[7] R. Machleidt, Adv. Nucl. Phys. 19, 189 (1989)

[8] B. ter Haar and R. Malfliet, Phys. Rep. 149, 207 (1987) 